\title{
Impurity induced non-bulk stacking in chemically exfoliated h-BN nanosheets
}

Cite this: Nanoscale, 2013, 5, 2290

\author{
Aleksey Shmeliov, ${ }^{a}$ Judy S. Kim, ${ }^{a}$ Konstantin B. Borisenko, ${ }^{\text {ae }}$ Peng Wang, ${ }^{b}$ \\ Eiji Okunishi, ${ }^{c}$ Mervyn Shannon, ${ }^{d}$ Angus I. Kirkland, ${ }^{\text {ae }}$ Peter D. Nellist ${ }^{a}$ \\ and Valeria Nicolosi ${ }^{\star f}$
}

Received 29th October 2012

Accepted 2nd February 2013

DOI: $10.1039 / c 3 n r 33375 b$

www.rsc.org/nanoscale

Structural characterization of 2D nanomaterials is an important step towards their future applications. In this work we carried out imaging and structural analysis of 2D h-BN produced by chemicalexfoliation, emphasizing the stacking order in few-layer sheets. Our analysis, for the first time has shown conclusively that non-bulk stacking can exist in 2D h-BN.

Compounds with layered crystal structure are an emergent and diverse source of two-dimensional nanomaterials with high specific surface areas that are important for applications in sensing, ${ }^{1}$ catalysis, ${ }^{2}$ energy harvesting ${ }^{3}$ and storage. ${ }^{4}$ Whilst graphene is the most renowned, there is considerable interest in other layered materials such as $2 \mathrm{D}$ hexagonal boron nitride (h-BN). Electronically, h-BN is a dielectric with direct bandgap of approximately $5.8 \mathrm{eV}^{5}$ making it a candidate for applications as a substrate in graphene-based field effect transistors ${ }^{6,7}$ and a top-gate dielectric. ${ }^{8}$ Chemical exfoliation methods of bulk h-BN has been shown to produce useful quantities of monolayer and stacked bi- and few-layer ${ }^{9}$ and a rapid growth in the technological applications of these is expected to follow. For these applications the structural properties of the material as synthesised will play an important role in determining the intrinsic properties and subsequent performance in devices.

As we examine the structure and stacking of h-BN, it is important to note the possible high-symmetry structural

\footnotetext{
${ }^{a}$ University of Oxford, Department of Materials, Parks Road, OX1 3PH, Oxford, UK ${ }^{b}$ National Laboratory of Solid State Microstructures, Department of Materials Science and Engineering, Nanjing 210093, People's Republic of China

${ }^{c} E M$ Application Group, EM Business UNIT, JEOL Ltd., 1-2 Musasino 3-chome, Akishima, Tokyo 196-8558, Japan

${ }^{d}$ SuperSTEM, STFC Daresbury Laboratories, Keckwick Lane, Warrington, WA4 4AD, UK ${ }^{e}$ Research Complex at Harwell, Rutherford Appleton Laboratory, Harwell Oxford, Didcot OX11 OFA, UK

${ }^{f}$ School of Chemistry, School of Physics \& CRANN, Trinity College Dublin, Dublin 2, Ireland.E-mail: nicolov@tcd.ie
}

$\dagger$ Electronic supplementary information (ESI) available. See DOI: 10.1039/c3nr33375b models projected along the [001] and [110] directions based on variations of both "simple" and "Bernal" stacking often reported in the literature for h-BN ${ }^{\mathbf{1 0 - 1 2}}$ (Scheme 1). These include: $\mathrm{AA}^{\prime}$ stacking, usually found in the bulk h-BN material, where atomic columns projected along the [001] direction consist of alternating $\mathrm{B}$ and $\mathrm{N}$ atoms (Scheme 1a); AA stacking where monolayer structure repeats itself; and two other high symmetry configurations $\mathrm{AB}$ and $\mathrm{ABC}$ (Scheme $1 \mathrm{c}$ and $\mathrm{d}$ respectively) formed by shifting the h-BN layers by $1 / 3$ and $1 / 3,2 / 3$ a unit cell as compared to the AA, respectively.

Theoretical calculations indicate similar cohesive energies for all these models ${ }^{\mathbf{1 0 - 1 2}}$ where the AA type (which has not been observed experimentally in pure h-BN) is calculated as least energetically favourable. ${ }^{12}$ Moreover, $\mathrm{AB}$ stacking was shown to be the most stable for h-BN bilayers. ${ }^{11}$ Van der Waals forces have been predicted to determine the interlayer distance and electrostatic forces have been shown to dictate the optimal stacking sequence. ${ }^{13}$ This suggests that modifications of stacking arrangements may occur through intercalation of ionic species between the layers and at the edges. In fact, a theoretical calculations of h-BN intercalated with neutral potassium suggest the AA stacking between the h-BN layers. ${ }^{\mathbf{1 4}}$

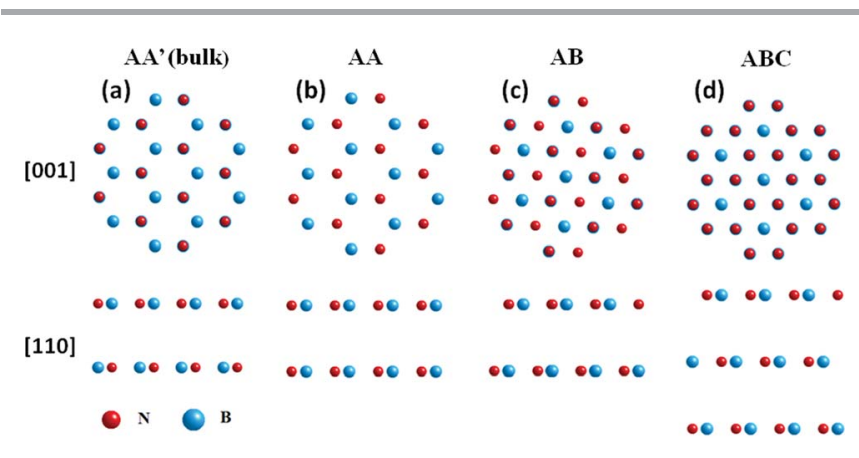

Scheme 1 h-BN structural models. Views along [001] show the projected structures that are recorded in the experimental ADF images. Views along [110] unambiguously show the alternative stacking sequences. The interlayer distance of [110] is shown to scale with respect to the interatomic distances of the [001] view. 
To-date, the majority of structural studies on $2 \mathrm{D}$ h-BN have focused on the edge structures, topological, point, and substitutional defects, ${ }^{15-20}$ largely ignoring stacking sequence and their local variations. In this paper we have investigated the stacking sequences present in sheets of h-BN produced by liquid-phase exfoliation of bulk h-BN in common organic solvents. ${ }^{9}$ We have used a combination of high spatial resolution aberration-corrected electron microscopy in both Annular Dark Field (ADF) Scanning Transmission Electron Microscopy (STEM) and phase contrast TEM imaging modes together with exit-wave reconstruction, electron diffraction and theoretical quantum chemical calculations.

ADF STEM has been effective in the study of $2 \mathrm{D}$ h-BN nanosheets ${ }^{19}$ as the incoherent nature of ADF STEM provides direct atomic imaging without contrast reversals at changing defocus levels. More importantly, the relatively high angles of electron scattering involved in this imaging mode result in image intensities strongly dependent upon atomic number. ${ }^{21}$ This enables direct observation and identification of $\mathrm{B}$ and $\mathrm{N}$ sub-lattices, as well as substitutional defects.

The use of exit wave reconstruction from a focal series of high resolution TEM images has the advantage of reducing Poisson image noise and eliminating delocalisation effects and other degradations arising from aberrations in the objective lens. ${ }^{22,23}$ Moreover, a real space image phase plate can be applied to the exit-wave post-reconstruction for local compensation of the measured uncorrected residual aberrations.

In order to determine the stacking sequences present, which requires segregation of the $\mathrm{B}$ and $\mathrm{N}$ sub-lattices we have performed a quantitative analysis of the atomic column intensities. In the atomic resolution ADF STEM image of a typical h-BN nanosheet (Fig. 1a), examination of the relative intensity profile (Fig. 1b) and histogram of the column intensities (Fig. 1c) allow an accurate determination of the two regions corresponding to the discreet number of layers. This has enabled us to calculate the mean column intensities in the $\mathrm{B}$ and $\mathrm{N}$ sub-lattices for each of these two regions (Table 1 ). In region 1 , the average peak intensity of the $\mathrm{N}$ atoms is higher than the average peak

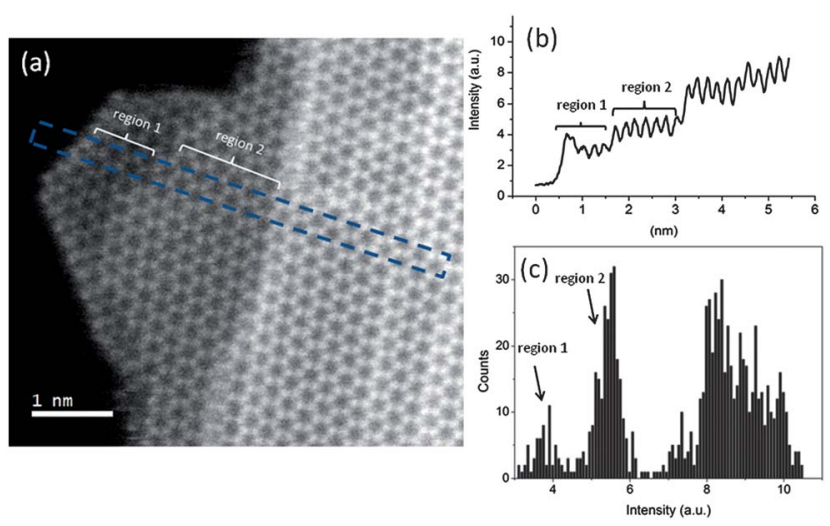

Fig. 1 ADF STEM image of h-BN nanosheet with supplementary graphs used for the stacking analysis. (a) ADF STEM image of h-BN sheet. (b) Intensity profile from the marked region. (c) Histogram of the column intensities. The image in (a) was filtered using a Butterworth low-pass filter. Integration width in (b) is $0.33 \mathrm{~nm}$. The values in (c) are referenced to the vacuum level.
Table 1 Statistical analysis of the nearest neighbour columns (B and N sites) from the two thinnest regions marked in Fig. 1a. Sites B and N correspond to the 2 basis defining the structure of the monolayer. For the bilayer $\mathrm{B}$ and $\mathrm{N}$ sites are consistently defined relative to the monolayer by the translational symmetry. The averaged values for both regions are given. Mean intensity values are referenced to the vacuum level

\begin{tabular}{lllll}
\hline & $\begin{array}{l}\text { Region 1 } \\
(\text { site B) }\end{array}$ & $\begin{array}{l}\text { Region 1 } \\
(\text { site N) }\end{array}$ & $\begin{array}{l}\text { Region 2 } \\
\text { (site B) }\end{array}$ & $\begin{array}{l}\text { Region 2 } \\
\text { (site N) }\end{array}$ \\
\hline $\begin{array}{l}\text { Mean } \\
\text { intensity } \\
\begin{array}{l}\text { Mean } \\
\text { intensity }\end{array}\end{array}$ & $2.75 \pm 0.04$ & $3.06 \pm 0.04$ & $4.54 \pm 0.03$ & $4.50 \pm 0.03$ \\
\end{tabular}

intensity of the $\mathrm{B}$ atoms suggesting that this region is a monolayer. The intensity difference between nearest neighbour sites in a bilayer that follows an $\mathrm{AA}^{\prime}$ stacking sequence is expected to equalize. The data presented in Table 1 follows this pattern, confirming that region 2 is a bilayer and nanosheet has bulk stacking. It must be noted that for $Z$-contrast imaging one would expect much larger intensity difference between boron and nitrogen sites for monolayer region. The reason for such discrepancy is the contribution of the probe tail. ${ }^{19}$

Interestingly, the vacuum to-monolayer edge intensity change in Fig. 1 is nearly twice as high as that observed for the monolayer to-bilayer step-edge. This raises concern that region 1 may be a bilayer and region 2 a trilayer. However, a timed sequence of images of the h-BN nanosheet (ESI, Fig. S1†) shows atoms diffusing from the edges of the suggested monolayer and bilayer regions. Edge diffusion reveals underlying layers consistent with intensity of the region 1 at the step edge and more vacuum at the vacuum-crystal interface. This further supports that regions 1 and 2 are monolayer and bilayer, correspondingly. One explanation of the extra large vacuum tomonolayer edge intensity change may be the presence of mobile physisorbed impurities at the nanosheet's surface.

A small fraction (two out of approximately 20) of the high resolution $\mathrm{ADF}$ images did not show the honeycomb pattern (Fig. 1a). For example, the image in Fig. 2a displays several regions where the stacking sequence appears to change. Fig. $2 \mathrm{~b}$ shows a region where an additional atomic column is present in the centre of the honeycomb lattice. This new projection is similar to the patterns formed by $\mathrm{AB}$ and $\mathrm{ABC}$ structures (Schemes $1 \mathrm{~b}$ and $\mathrm{c}$ ). The $\mathrm{AB}$ stacking in h-BN was also identified indirectly by diffraction analysis (Fig. S2 $\dagger$ ) as described further in the ESI. Additionally, some of the atomic columns in Fig. $2 b$ appear displaced from their normal lattice positions with an apparent shear strain in the range of $4-12 \%$ (ESI, Fig. S3 $†$ ). By comparison, the shear required to form a stacking fault is $44 \%$.

Furthermore, in the region of interest (Fig. 2c) each additional layer results in an increase of the intensity difference between the nearest neighbour sites. Analysis of the atomic column intensities shows that the intensity difference between the nearest neighbour sites in this region is approximately equivalent to the product of the number of layers and the intensity difference measured in a monolayer. The averaged intensity difference between nearest neighbour sites were 

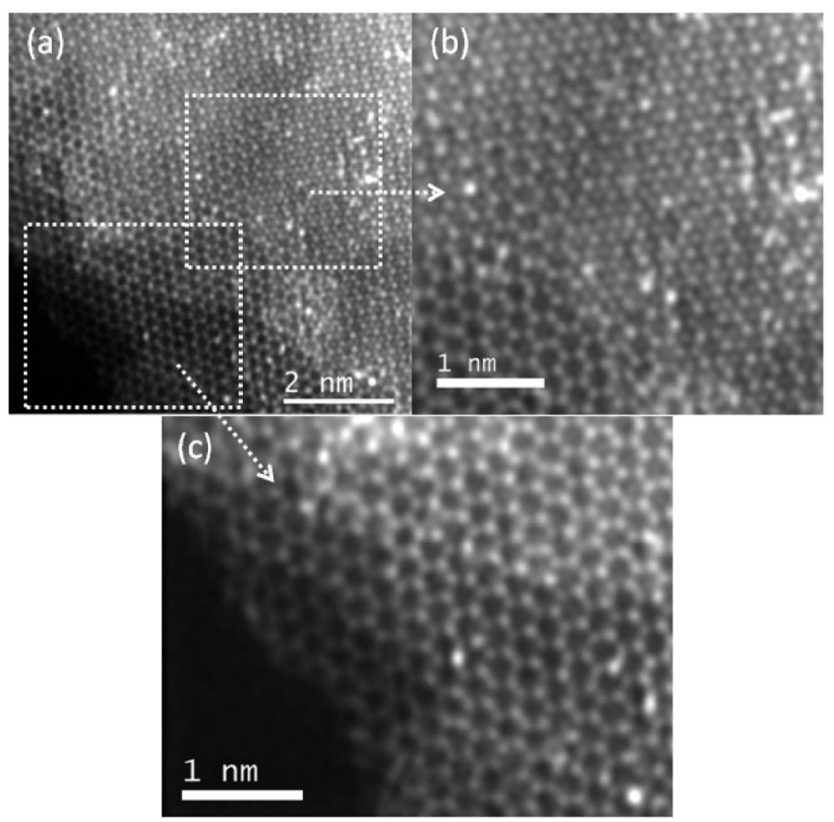

Fig. 2 (a) ADF STEM image of h-BN nanoflake. (b) and (c) magnified subregions of image (a). Image (a) was processed using a Gaussian bandpass filter. ${ }^{19}$

$1.02 \pm 0.62,1.85 \pm 0.90,2.88 \pm 0.70$, and $4.54 \pm 0.11$ a.u. for the mono-, bi-, tri-, and quadralayer respectively. This is consistent with simple AA stacking, in which B atoms are stacked above B atoms and $\mathrm{N}$ atoms are stacked above $\mathrm{N}$ atoms (Scheme $1 \mathrm{~b}$ ). However, it should be pointed out that due to the high level of noise in the images the errors are rather high and a computed statistical histogram of the column intensities did not show discrete peaks. Consequently, our counting of the number of layers in Fig. 2c was based upon an assessment of the visual contrast. This observation agrees with the quantum chemical calculations that suggest that AA stacking can be stabilised by adsorbed atoms (see ESI $\dagger$ ). There is additional concern that the observed intensity difference between nearest neighbour sites is caused by higher order non-round aberrations. Such possibility is discussed in details in ESI, $\uparrow$ concluding that not only would the size of the aberration need to be large enough, but it would also need to be aligned with the lattice. We therefore can conclude that the observed effect is not caused by the aberrations. A large number of impurity atoms appear as bright spots in Fig. 2a. A comparison of the atomic columns intensities at impurity sites with equivalent crystallographic sites, can give an estimate of the atomic number of the impurity atom species (ESI, Fig. S4†). Our analysis indicates $Z$ to lie in the range of 714. This is consistent with previous work ${ }^{19}$ where Na adatoms $(Z=11)$ were frequently found in h-BN nanosheets similarly exfoliated using the solvent normal methyl pyrrolidinone (NMP). Commercially available NMP typically contains traces of $\mathrm{Na}(\sim 100 \mathrm{ppb})$ and other amine impurities at about $12 \mathrm{ppm}$. Experimentally, non-bulk staking was only found in nanoflakes contaminated by impurities. It is therefore possible that this relatively high concentration of impurities stabilises the abnormal stacking sequences observed that are not energetically favourable in the pristine state.
In order to further verify this hypothesis we have carried out a pilot experiment in which $\mathrm{Na}^{+}$species (in the form of $\mathrm{NaCl}$ ) were deliberately added to the exfoliated nanosheets in solution. Electron diffraction was used to assess the stacking of the exfoliated 2D material (ESI, Fig. S2.2†). For this purpose a few drops of h-BN dispersions with and without $\mathrm{NaCl}$ addition, were vacuum filtrated onto holey carbon TEM grids in order to obtain restacked nanofilms with uniform coverage. Thirty diffraction patterns were recorded for the as-exfoliated h-BN and the $\mathrm{Na}-\mathrm{h}$ BN samples respectively. From this we obtained the averaged ratio of the $\{10 \overline{1} 0\}$ and $\{11 \overline{2} 0\}$ diffraction ring intensities, $I_{\{10 \overline{1} 0\}} /$ $I_{\{11 \overline{2} 0\}}$. The ratios were $1.18 \pm 0.04$ for the as-exfoliated h-BN sheets and $0.89 \pm 0.02$ for the $\mathrm{Na}-\mathrm{h}-\mathrm{BN}$ sheets. This difference in ring intensity ratios was consistent and reproducible for the large number of diffraction patterns acquired, indicating a substantial change to the stacking sequence caused by the addition of $\mathrm{Na}^{+}$to the h-BN dispersions. A comparison between the calculated (ESI, Table $\mathrm{S} 1 \dagger$ ) and the experimental ratios suggests that introduction of $\mathrm{NaCl}$ causes stacking faults, similar in structure to $\mathrm{AB}$ and/or $\mathrm{ABC}$ structures, having the overall effect of lowering the diffraction rings' intensity ratios.

Quantum chemical calculations on h-BN bi-layer sheets have shown that the presence of $\mathrm{Na}^{+}$ions adsorbed at the edges of
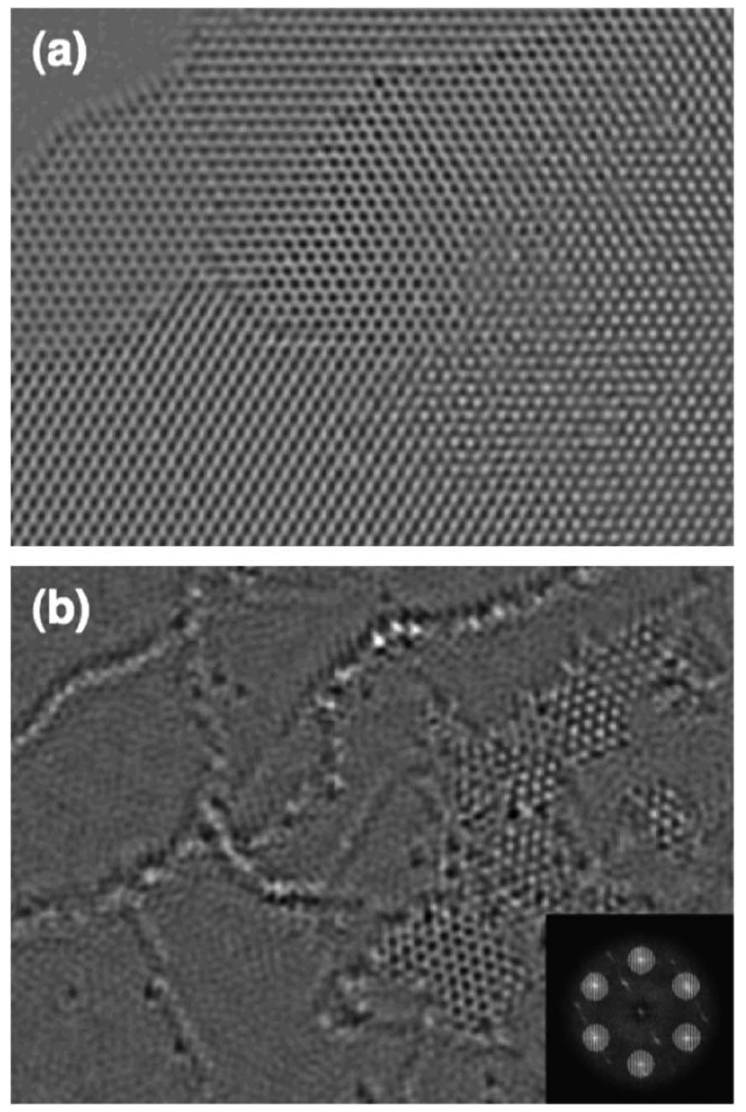

Fig. 3 Exit-wave reconstructed images of the h-BN nanosheet. (a) Phase of the reconstructed exit-wave of a thin h-BN membrane. (b) Phase of the same region after filtering away the primary lattice reflections with a mask (inset). Localised regions of the hexagonal lattice remain in regions where they are oriented with a $30^{\circ}$ rotation to the main lattice. 
h-BN sheets can also have the effect of rotating the layers relative to each other by approximately $20^{\circ}$ (see ESI $\dagger$ ). A similar observation on a somewhat larger scale was observed in the phase of the specimen exit wave restored from a focal series of high resolution TEM images (Fig. 3). Fig. 3a shows the reconstructed phase of a thin h-BN flake, revealing the typical hexagonal lattice expected for the [001] projection. Fig. 3b shows the reconstructed phase of the same region after removal of the main lattice reflections by Fourier filtering. This analysis reveals interesting stacking information, clearly indicating a few regions of the nanosheet that are rotated at $\mathrm{ca} .30^{\circ}$ with respect to the primary lattice. It is also interesting to note the presence of small bright features dotted along the step edges (Fig. 3b) which represent a larger phase shift. These are likely due to a presence of heavier atomic species that stabilise the rotation, consistent with the calculations.

\section{Conclusions}

This work has described experimental confirmation of stacking variations in 2D h-BN for the first time. On the basis of quantitative analysis of the ADF STEM atomic column intensities we have shown that majority of h-BN nanosheets exhibit the $\mathrm{AA}^{\prime}$ stacking sequence observed in the bulk. However, we have also observed alternative stacking sequences in which additional atomic columns are present in projection inside the honeycomb lattice forming features consistent with $\mathrm{AB}$ or $\mathrm{ABC}$ sequences. AA stacking, which was previously predicted to be energetically unfavourable, was also identified within a small region of a nanoflake. Electron diffraction intensities provided further evidence for the existence of a non-bulk stacking in 2D h-BN. Furthermore, our data also suggests that non-bulk stacking in $2 \mathrm{D}$ h-BN is strongly influenced by the presence of impurities. Quantum calculations were used as a guide to predict possible variations in the stacking sequence due to sodium ion adsorption, and it was experimentally observed that the presence of the sodium dopants can facilitate rotations of the layers in $\mathrm{AA}^{\prime}$ stacked bilayers. These stacking deviations are expected to substantially affect the physical chemical and electronic properties of the material. Our finding that the stacking sequence can be tuned by doping with impurity species such as $\mathrm{Na}$ becomes an attractive option for control of these properties with important consequences for material application.

\section{Experimental methods}

Dispersions were produced by ultra-sonication (UP $100 \mathrm{H}$ Ultrasonic Processor, Hielscher, 100\% amplitude, continuous cycle) of the bulk h-BN powder in either NMP or isopropanol for $2 \mathrm{~h}$. During ultra-sonication dispersions were placed in an icebath. After ultra-sonication dispersions were left to settle for over two nights. Finally supernatant was carefully decanted and samples were prepared by drop-casting onto Agar holey-carbon/ copper grids (500 mesh).

Bulk hexagonal boron nitride powders were provided by Saint-Gobain Advanced Ceramics Lauf $\mathrm{GmbH}$ with the stated chemical purity $>98 \%$ h-BN, $<1.5 \% \quad \mathrm{O}_{2},>0.5 \% \quad \mathrm{~B}_{2} \mathrm{O}_{3}$. NMP anhydrous and isopropanol were purchased from SigmaAldrich and used as received.

Conventional TEM and electron diffraction was carried out using JEOL2010 microscope operated at $200 \mathrm{keV}$.

For the ADF STEM the samples were preliminary baked for 3 $\mathrm{h}$ at $135{ }^{\circ} \mathrm{C}$. Imaging was done using JEM-ARM200F STEM operated at $80 \mathrm{keV}$ with a probe current of $\sim 0.2 \mathrm{nA}$. The collection half angle for electrons was 45-170 mrad (Fig. 2) and 55-170 mrad (Fig. 3).

The collection half angle for electrons was $45-170 \mathrm{mrad}$ (Fig. 1) and 55-170 mrad (Fig. 2). HRTEM images used for focal series exit wave reconstructions were collected using the double aberration corrected JEOL 2200MCO instrument at University of Oxford. The instrument was operated at $80 \mathrm{kV}$ with spherical aberration set to $\mathrm{C} 3=-3 \mu \mathrm{m}$ in order to partially offset residual C5 aberration effects. Remaining aberrations higher than the $3^{\text {rd }}$ order that could not be corrected in the instrument were removed from the exit wave post- experimentally using a phase plate.

\section{Acknowledgements}

VN wishes to thank the European Research Council for funding through the grant 2DNanoCaps.

\section{Notes and references}

1 F. Schedin, A. K. Geim, S. V. Morozov, E. W. Hill, P. Blake, M. I. Katsnelson and K. S. Novoselov, Nat. Mater., 2007, 6, 652-655.

2 F. A. Frame and F. E. Osterloh, J. Phys. Chem. C, 2010, 114, 10628-10633.

3 H. Tributsch, Sol. Energy Mater., 1979, 1, 10-12.

4 J. Xiao, D. Choi, L. Cosimbescu, P. Koech, J. Liu and J. P. Lemmon, Chem. Mater., 2010, 22, 4522-4524.

5 K. Watanabe, T. Taniguchi and H. Kanda, Phys. Status Solidi A, 2004, 201, 2561-2565.

6 C. R. Dean, A. F. Young, I. Meric, C. Lee, L. Wang, S. Sorgenfrei, K. Watanabe, T. Taniguchi, P. Kim, K. L. Shepard, et al., Nat. Nanotechnol., 2010, 5, 722-726.

7 R. Decker, Y. Wang, V. W. Brar, W. Regan, H.-Z. Tsai, Q. Wu, W. Gannett, A. Zettl and M. F. Crommie, Nano Lett., 2011, 11, 2291-2295.

8 I. Meric, C. Dean, A. Young, J. Hone, P. Kim and K. L. Shepard, in Electron Devices Meeting (IEDM), 2010 IEEE International, pp. 23.2.1-23.2.4.

9 J. N. Coleman, M. Lotya, A. O'Neill, S. D. Bergin, P. J. King, U. Khan, K. Young, A. Gaucher, S. De, R. J. Smith, et al., Science, 2011, 331, 568-571.

10 N. Ooi, A. Rairkar, L. Lindsley and J. B. Adams, J. Phys.: Condens. Matter, 2006, 18, 97-115.

11 R. Ribeiro and N. Peres, Phys. Rev. B: Condens. Matter Mater. Phys., 2011, 83, 1-6.

12 L. Liu, Y. Feng and Z. Shen, Phys. Rev. B: Condens. Matter Mater. Phys., 2003, 68, 1-8.

13 N. Marom, J. Bernstein, J. Garel, A. Tkatchenko, E. Joselevich, L. Kronik and O. Hod, Phys. Rev. Lett., 2010, 105, 1-4. 
14 S. Okada and M. Otani, Phys. Rev. B: Condens. Matter Mater. Phys., 2010, 81, 3-6.

15 W.-Q. Han, L. Wu, Y. Zhu, K. Watanabe and T. Taniguchi, Appl. Phys. Lett., 2008, 93, 223103.

16 C. Jin, F. Lin, K. Suenaga and S. Iijima, Phys. Rev. Lett., 2009, 102, 3-6.

17 N. Alem, R. Erni, C. Kisielowski, M. Rossell, W. Gannett and A. Zettl, Phys. Rev. B: Condens. Matter Mater. Phys., 2009, 80, $1-7$.

18 J. C. Meyer, A. Chuvilin, G. Algara-Siller, J. Biskupek and U. Kaiser, Nano Lett., 2009, 9, 2683-2689.
19 O. L. Krivanek, M. F. Chisholm, V. Nicolosi, T. J. Pennycook, G. J. Corbin, N. Dellby, M. F. Murfitt, C. S. Own, Z. S. Szilagyi, M. P. Oxley, et al., Nature, 2010, 464, 571-574.

20 J. S. Kim, K. B. Borisenko, V. Nicolosi and A. I. Kirkland, ACS Nano, 2011, 5, 3977-3986.

21 M. Treacy and A. Howie, Philos. Mag. A, 1978, 38, 569585.

22 H. W. Zandbergen and D. Van Dyck, Microsc. Res. Tech., 2000, 49, 301-323.

23 A. I. Kirkland and R. R. Meyer, Microsc. Microanal., 2004, 10, 401-413. 